Institut für Arbeitsmedizin, Sozialmedizin und Umweltmedizin der Goethe-Universität Frankfurt am Main

\title{
MERS-CoV: Ein aktueller Überblick
}

\author{
Matthias Bundschuh, Doris Klingelhoefer
}

Matthias Bundschuh, Doris Klingelhoefer: MERS-CoV: Ein aktueller Überblick. Zbl Arbeitsmed 63 (2013) 296-297

Schlüsselwörter: neues Coronavirus, MERS-CoV, Middle East Respiratory Syndrome

\section{Zusammenfassung:}

Der folgende Artikel gibt eine aktuelle Übersicht zu dem neu aufgetretenen Middle East Respiratory Syndrome Coronavirus (MERS-CoV). Erstmals im Sommer 2012 kam es in Ländern der arabischen Halbinsel zu schweren Infektionen der Atemwege durch ein neues Coronavirus (MERS-CoV). Dabei wurden die meisten Erkrankungsfälle aus Saudi-Arabien gemeldet, in Deutschland wurden bisher mehrere importierte Krankheitsfälle berichtet.

Nach Beginn mit grippeähnlichen Symptomen folgt meist die Entwicklung einer Pneumonie mit Übergang in ein akutes Atemnotsyndrom. Die Letalitätsrate wird auf 55\% geschätzt. Über die Erkrankung sollte im Rahmen der reisemedizinischen Beratung ausführlich aufgeklärt werden und sie ist insofern auch für die Arbeitsmedizin von großer Bedeutung.

\section{MERS-CoV: A current review}

Matthias Bundschuh, Doris Klingelhoefer: MERS-CoV: A current review. Zbl Arbeitsmed 63 (2013) 296-297

Key words: novel coronavirus, MERS-CoV, Middle East Respiratory Syndrome

Summary:

The following article gives a current overview of the newly emerging Middle East Respiratory Syndrome Coronavirus (MERS$\mathrm{CoV})$. Since first time in summer 2012 in some countries in the Arabian Peninsula there were a number of severe infections of the respiratory tract caused by the new coronavirus. The majority of cases of illness were reported from Saudi Arabia, in Germany several imported cases of the disease have been reported. After beginning with flu-like-symptoms usually follows a pneumonia with a transition to an acute respiratory distress syndrome. The mortality rate is estimated at 55\%. In the travel medical advice it should be elucidated about the disease and for that reason it is of great importance also for occupational medicine.

\section{Aktuelles}

Seit dem Sommer 2012 kam es in Ländern der arabischen Halbinsel vereinzelt zu einem Auftreten von schweren Infektionen der Atemwege durch ein neues Coronavirus (MERS-CoV). Ein Jahrzehnt nach der SARS-Pandemie (2002/2003) kam es somit erneut zu einer Atemwegsinfektion durch ein Virus aus der Familie der Coronaviren. Laut Angaben des Robert Koch-Institutes (RKI) gab es in bisher allen aufgetretenen Fällen eine Verbindung zu Patienten mit Herkunft von der arabischen Halbinsel. So kam es vor allem in Saudi-Ara- bien, aber auch Jordanien, Katar und den Vereinigten Arabischen Emiraten zum Auftreten dieser Erkrankung. Aus den europäischen Ländern Deutschland, Großbritannien, Tunesien, Frankreich und Italien wurden importierte Krankheitsfälle bzw. Sekundärfälle gemeldet [1]. Der Weltgesundheitsorganisation (WHO) wurden im Zeitraum von September 2012 bis September 2013 weltweit insgesamt 108 laborchemisch bestätigte Erkrankungsfälle durch MERSCoV gemeldet. Davon seien 50 Fälle letal verlaufen [2].

\section{MERS-Coronavirus}

Das MERS-Coronavirus aus der Familie der Coronaviren ist ein einzelsträngiges RNA-Virus mit einer Genomlänge von ca. 27.600 bis $31.000 \mathrm{Nu}$ kleotiden. Aufgrund von phylogenetischen Eigenschaften werden die Coronaviren in zwei verschiedene Unterfamilien und mehrere Gattungen differenziert, wobei die Spezies MERS-CoV der Gattung Betacoronavirus zugeordnet wird. Die Coronaviren sind genetisch hochvariabel und besitzen die längsten Genome aller bekannten RNA-

Kontaktadresse

Matthias Bundschuh

Zentrum für Gesundheitswissenschaften Institut für Arbeitsmedizin, Sozialmedizin und Umweltmedizin

Geschäftsführender Zentrumsdirektor: Prof. Dr. Dr. h.c. mult. D. Groneberg

Goethe-Universität Theodor-Stern-Kai 7 Haus $9 \mathrm{~b}$ - 60590 Frankfurt am Mai

Tel.: +49696301-4364 Fax : +49696301-7053_E-Mail: Bundschuh@med.uni-frankfurt.de 
Tabelle 1: Erkrankungs- und Todesfälle durch MERS-CoV (modifiziert nach [3])

\begin{tabular}{|l|l|l|}
\hline Länder & Erkrankungsfälle & Todesfälle \\
\hline Frankreich & 2 & 1 \\
\hline Italien & 3 & 0 \\
\hline Jordanien & 2 & 2 \\
\hline Katar & 4 & 1 \\
\hline Saudi Arabien & 86 & 42 \\
\hline Tunesien & 2 & 0 \\
\hline Großbritannien & 3 & 2 \\
\hline Vereinigte Arabische Emirate & 6 & 2 \\
\hline Insgesamt & 108 & 50 \\
\hline
\end{tabular}

Viren $[4,5]$. Das komplette Genom dieses neuartigen Coronavirus wurde in dem Erasmus Medical Center (EMC) in Rotterdam sequenziert [5]. Die Vertreter der Coronaviren verursachen bei Wirbeltieren wie Säugetieren, Nagetieren, Fischen und Vögeln unterschiedliche Erkrankungen. Beim Menschen sind sie als Auslöser von leichten respiratorischen Infektionen bis hin zum schweren akuten Atemwegssyndrom bekannt [6].

\section{Reservoir und \\ Übertragungsmöglichkeiten}

Der genaue Übertragungsweg der Erkrankung ist bisher noch unbekannt. Aufgrund einer engen genetischen Verwandtschaft mit den bei Fledermäusen vorkommenden Coronaviren werden als Reservoir für die MERS-CoV vor allem Fledermausspezies aus Südafrika diskutiert, jedoch gibt es dafür bislang noch keinen endgültigen Nachweis [6]. Auch weitere Tierspezies sind als Reservoir nicht auszuschließen. Mittlerweile wurden auch einzelne Mensch-zu-Mensch Übertragungen dokumentiert und traten vor allem bei Familienangehörigen, medizinischem Personal und im Arbeitsumfeld der Patienten auf. Der genaue Übertragungsweg auf den Menschen, also ob im Rahmen einer Tröpfcheninfektion durch Husten oder Niesen oder durch direkten Körperkontakt ist bisher nicht bekannt.

\section{Klinik und Diagnostik}

Die Erkrankung beginnt mit einer akut auftretenden, grippeähnlichen Symptomatik, im weiteren Verlauf folgt häufig eine Pneumonie und anschließend in einigen Fällen der Übergang in ein akutes Atemnotsyndrom Einige Patienten hatten eine gastrointestinale Begleitsymptomatik mit Diarrhoe. Auch akutes Nierenversagen wird beschrieben [7]. Besonders gefährdet sind chronisch vorerkrankte Patienten, wie z.B. onkologische, immunsupprimierte Patienten sowie Diabetiker. Differentialdiagnostisch sollte an eine MERS-CoV-Infektion gedacht werden, wenn der Patient 14 Tage vor Erkrankungsbeginn in einem Land der Arabischen Halbinsel unterwegs gewesen ist oder Kontakt mit einem $\mathrm{Pa}$ tienten mit nachgewiesener oder anzunehmender MERS-CoV-Infektion hatte. Die WHO empfiehlt bei entsprechendem Verdacht auf eine MERS-CoVInfektion die Entnahme von Proben aus dem unteren Respirationstrakt (Sputum, Trachealaspirat, bronchoalveoläre Lavage). Die spezifische Diagnostik des neuartigen Coronavirus ist am Robert KochInstitut und am Institut für Virologie der Universität Bonn etabliert. Grundsätzlich sollte bei schweren Pneumonien und Atemnotsyndrom immer eine labordiagnostische Abklärung erfolgen, auch wenn die Reiseanamnese negativ erscheint $[1,6]$.

\section{Therapie}

Eine spezifische antivirale Therapie ist bisher wie auch eine Impfung nicht vorhanden, die Behandlung erfolgt supportiv [6].

\section{Bedeutung in der Arbeitsmedizin}

Wie berichtet, hat die Transmission des MERS-CoV von der Arabischen Halbinsel auf den europäischen Kontinent bereits stattgefunden. Aufgrund eines immer mehr zunehmenden globalen Tourismus ist es vorstellbar, dass es zu einer weltweiten Ausbreitung kommt, wenn beispielsweise infizierte Patienten aus medizinischen Gründen ihre Heimatländer verlassen. Insofern sollte jeder praktisch tätige Arzt sowie Arbeitsmediziner diesbezüglich sensibilisiert sein und bei Patienten mit dem klinischen Bild eines akuten Atemnotsyndroms und positiver Reiseanamnese differentialdiagnostisch eine Infektion durch das MERS-CoV in Betracht ziehen.

\section{Literatur:}

1. RKI: 2013: Informationen des RKI zu Erkrankungsfällen durch das Middle East Respiratory Syndrome Coronavirus (MERS-CoV). In: http://www.rki.de/DE/Content/InfAZ/C/ Corona/MERS-CoV.html;jsessionid= 29336F9294B573E73ABAA7EAA46A8196. 2 cid372? nn=3223662.

2. WHO: 2013. Middle East respiratory syndrome coronavirus (MERS-CoV) - update. In: http://www.who.int/csr/don/2013_08_30/en/ index.html. .

3. CDC: 2013: MERS. In: http://www.cdc.gov/ coronavirus/mers/.

4. Al-Tawfiq, Jaffar A. Middle East Respiratory Syndrome-coronavirus infection: An overview. J Infect Public Health, 2013 Jul 26.

5. Geng, H.: A novel human coronavirus: Middle East respiratory syndrome human coronavirus. Sci China Life Sci, 2013, August;56 (8):683-7.

6. WHO: 2013: Frequently Asked Questions on Middle East respiratory syndrome coronavirus (MERS-CoV). In: http://www.who.int/csr/ disease/coronavirus_infections/faq/en/.

7. AlBarrak AM, Stephens GM, Hewson R, Memish ZA: Recovery from severe novel coronavirus infection. Saudi Medical Journal 2012, 33:1265-1269. 